\title{
Local boundedness of the number of solutions of Plateau's problem for polygonal boundary curves
}

\section{Journal Article}

Author(s):

Jakob, Ruben

Publication date:

2008

Permanent link:

https://doi.org/10.3929/ethz-b-000422489

Rights / license:

In Copyright - Non-Commercial Use Permitted

Originally published in:

Annals of global analysis and geometry 33(3), https://doi.org/10.1007/s10455-007-9082-8 


\title{
Local boundedness of the number of solutions of Plateau's problem for polygonal boundary curves
}

\author{
Ruben Jakob
}

Received: 27 February 2007 / Accepted: 11 July 2007 / Published online: 8 August 2007

(C) Springer Science+Business Media B.V. 2007

\begin{abstract}
In the present article, the author proves two generalizations of his "finitenessresult" (I.H.P. Anal. Non-lineaire, 2006, accepted) which states for any extreme simple closed polygon $\Gamma \subset \mathbb{R}^{3}$ that every immersed, stable disc-type minimal surface spanning $\Gamma$ is an isolated point of the set of all disc-type minimal surfaces spanning $\Gamma$ w.r.t. the $C^{0}$-topology. First, it is proved that this statement holds true for any simple closed polygon in $\mathbb{R}^{3}$, provided it bounds only minimal surfaces without boundary branch points. Also requiring that the interior angles at the vertices of such a polygon $\Gamma$ have to be different from $\frac{\pi}{2}$ the author proves the existence of some neighborhood $O$ of $\Gamma$ in $\mathbb{R}^{3}$ and of some integer $\beta$, depending only on $\Gamma$, such that the number of immersed, stable disc-type minimal surfaces spanning any simple closed polygon contained in $O$, with the same number of vertices as $\Gamma$, is bounded by $\beta$.
\end{abstract}

Keywords Local boundedness of the number of solutions · Plateau's problem for polygonal boundary curves

Mathematics Subject Classification 49Q05

\section{Introduction and main result}

In [14] the author proved for any extreme simple closed polygon $\Gamma \subset \mathbb{R}^{3}$ that every immersed, stable disc-type minimal surface spanning $\Gamma$ is an isolated point of the set of all disc-type minimal surfaces spanning $\Gamma$ w.r.t. the $C^{0}$-topology. Here a polygon is termed extreme, if it is contained in the boundary of some convex compact subset of $\mathbb{R}^{3}$. The aims of the present article are proofs of the following two generalizations:

Theorem 1.1 Let $\Gamma \subset \mathbb{R}^{3}$ be an arbitrary simple closed polygon which meets the requirement to bound only minimal surfaces without boundary branch points. Then every immersed,

R. Jakob $(\varangle)$

ETHZ, Rämistr. 101, 8092 Zurich, Switzerland

e-mail: ruben.jakob@math.ethz.ch 
stable disc-type minimal surface spanning $\Gamma$ is an isolated point of the set of all disc-type minimal surfaces spanning $\Gamma$ w. r. t. the $C^{0}$-topology. In particular, $\Gamma$ can bound only finitely many immersed, stable disc-type minimal surfaces.

Theorem 1.2 Let $\Gamma^{*} \subset \mathbb{R}^{3}$ be an arbitrary polygon with $N+3>3$ vertices meeting the requirements of Theorem 1.1, and the additional one that the interior angles at its vertices are different from $\frac{\pi}{2}$. Then, there exists some neighborhood $O$ of $\Gamma^{*}$ in $\mathbb{R}^{3}$ and some integer $\beta$, depending only on $\Gamma^{*}$, such that the number of immersed, stable disc-type minimal surfaces spanning any simple closed polygon $\Gamma \subset O$ also with $N+3$ vertices is bounded by $\beta$.

A disc-type minimal surface $X$ is called immersed if there holds $\inf _{B}|D X|>0$, where we have denoted by $B$ the open unit disc $\left\{w=(u, v) \in \mathbb{R}^{2} \| w \mid<1\right\}$. It is said to be stable if the second variation of the area functional $\mathcal{A}$ in $X$ in normal direction $\xi:=\frac{X_{u} \wedge X_{v}}{\left|X_{u} \wedge X_{v}\right|}$

$$
J^{X}(\varphi):=\int_{B}|\nabla \varphi|^{2}+2 K E \varphi^{2} d w=\left.\frac{d^{2}}{d \epsilon^{2}} \mathcal{A}(X+\epsilon \varphi \xi)\right|_{\epsilon=0}
$$

satisfies $J^{X}(\varphi) \geq 0$ for any $\varphi \in C_{c}^{\infty}(B)$, where $E$ denotes $\left|X_{u}\right|^{2}$ and $K \leq 0$ the Gauss curvature of $X$.

We shall compare Theorem 1.2 to Tomi's result in [19]:

Theorem 1.3 [19] If $\mathcal{R}$ is an element of the set $\mathcal{P}$ of proper curves, then $\mathcal{M}_{s}(\mathcal{R})$ is finite. Moreover, there exists some $C^{4, \alpha}$-neighborhood $O$ of $\mathcal{R}$ in $\mathcal{P}$ and some integer $\beta$, depending only on $\mathcal{R}$, such that the number of immersed, stable disc-type minimal surfaces spanning any proper curve contained in $O$ is bounded by $\beta$.

Here the set $\mathcal{P}$ of proper curves consists of regular Jordan curves in $\mathbb{R}^{3}$ of class $C^{4, \alpha}$ which bound only minimal surfaces without boundary branch points and with interior branch points of at most first order.

\section{Basic definitions and tools}

A polygon $\Gamma \subset \mathbb{R}^{3}$ is a closed piecewise linear Jordan curve being determined by the positions of its $N+3$ vertices $(N \in \mathbb{N})$

$$
\left(P_{1}, P_{2}, \ldots, P_{N+3}\right)=: P \in \mathbb{R}^{3 N+9},
$$

where we require the pairs of vectors $\left(P_{j+1}-P_{j}, P_{j}-P_{j-1}\right)$ to be linear independent for $j=1, \ldots, N+3$, with $P_{0}:=P_{N+3}$ and $P_{N+4}:=P_{1}$. Thus, we have a correspondence $P \longleftrightarrow \Gamma(P)$ between polygons in $\mathbb{R}^{3}$ satisfying the above requirements and "admissible" vertex tuples $P \in \mathbb{R}^{3 N+9}$. We consider the "Plateau class" $\mathcal{C}^{*}(\Gamma)$ of surfaces $X \in$ $H^{1,2}\left(B, \mathbb{R}^{3}\right) \cap C^{0}\left(\bar{B}, \mathbb{R}^{3}\right)$ that are spanned into $\Gamma$, i.e., whose boundary values $\left.X\right|_{\partial B}$ : $\mathbb{S}^{1} \longrightarrow \Gamma$ are weakly monotonic mappings with degree equal to 1 , satisfying a three-point condition: $X\left(e^{i \tau_{N+k}}\right)=P_{N+k}$ for $\tau_{N+k}:=\frac{\pi}{2}(1+k), k=1,2,3$. We endow $\mathcal{C}^{*}(\Gamma)$ with the norm $\|\cdot\|_{C^{0}(\bar{B})}$ and denote by $\mathcal{M}(\Gamma)$ its subspace $\left\{X \in \mathcal{C}^{*}(\Gamma)|\triangle X=0,| X_{u}|=|\right.$ $X_{v} \mid,\left\langle X_{u}, X_{v}\right\rangle=0$ on $\left.B\right\}$ of disc-type minimal surfaces. Furthermore, let $\mathcal{M}_{s}(\Gamma)$ be the subspace of $\mathcal{M}(\Gamma)$ consisting of those elements which are immersed and stable. It should be mentioned that the Theorems 2 and 3 in [10] yield the existence of a global minimizer $X^{*}$ of $\mathcal{A}$ within $\mathcal{C}^{*}(\Gamma)$, i.e., of some $X^{*} \in \mathcal{C}^{*}(\Gamma)$ satisfying $\mathcal{A}\left(X^{*}\right)=\inf _{\mathcal{C}^{*}(\Gamma)} \mathcal{A}$, which 
is, furthermore, free of branch points on $B$ by Alt's papers [1] and [2]. If we require the polygon $\Gamma$ to bound only minimal surfaces without boundary branch points, as we do in the formulations of the Theorems 1.1 and 1.2, then $X^{*}$ is even immersed and thus an element of $\mathcal{M}_{s}(\Gamma)$. Hence, we infer from this observation:

Proposition 2.1 $\mathcal{M}_{S}(\Gamma)$ is nonvoid for any polygon $\Gamma$ as in Theorem 1.1.

Our fundamental tools are Courant's [3] and Heinz' [5, 6] maps

$$
\psi: T \longrightarrow\left(\mathcal{C}^{*}(\Gamma),\|\cdot\|_{C^{0}(\bar{B})}\right), \quad \tilde{\psi}: T \longrightarrow C^{0}\left(\bar{B}, \mathbb{R}^{3}\right) \cap C^{2}\left(B, \mathbb{R}^{3}\right),
$$

which are assigned to our arbitrarily fixed-closed polygon $\Gamma(P)$. Here $T$ is an open, bounded, and convex set of $N$-tuples $\left(\tau_{1}, \tau_{2}, \ldots, \tau_{N}\right)=: \tau \in(0, \pi)^{N}$, which meet the following chain of inequalities $0<\tau_{1}<\cdots<\tau_{N}<\pi=\tau_{N+1}$, where $N+3$ was the number of vertices of the considered polygon $\Gamma(P)$. Moreover, to any point $\tau \in T$ and the fixed vertex tuple $P$ we assign the sets of surfaces

$$
\begin{array}{r}
\mathcal{U}(P, \tau):=\left\{X \in \mathcal{C}^{*}(\Gamma)|X|_{\partial B}\left(e^{i \tau_{j}}\right)=P_{j} \text { for } j=1, \ldots, N\right\} \text { and } \\
\tilde{\mathcal{U}}(P, \tau):=\left\{X \in C^{0}\left(\bar{B}, \mathbb{R}^{3}\right) \cap C^{2}\left(B, \mathbb{R}^{3}\right) \mid X\left(e^{i \theta}\right) \in \Gamma_{j} \text { for } \theta \in\left[\tau_{j}, \tau_{j+1}\right]\right\},
\end{array}
$$

for $1 \leq j \leq N+3$, where we set $\Gamma_{j}(P):=\left\{P_{j}+t\left(P_{j+1}-P_{j}\right) \mid t \in \mathbb{R}\right\}, P_{N+4}:=P_{1}$ and $\tau_{N+4}:=\tau_{1}$. On account of two uniqueness results in [3] resp. [5] one can assign to the chosen vertex tuple $P$ the maps

$$
\begin{gathered}
\psi(P, \tau):=\text { unique minimizer of } \mathcal{D} \text { within } \mathcal{U}(P, \tau) \text { and } \\
\tilde{\psi}(P, \tau):=\text { unique minimizer of } \mathcal{D} \text { within } \tilde{\mathcal{U}}(P, \tau),
\end{gathered}
$$

where $\mathcal{D}$ denotes Dirichlet's integral. We will also use the notation $X(\cdot, P, \tau)$ for $\tilde{\psi}(P, \tau)$. Now by the result of [3] (see also [11], p. 558), Satz 1 in [5] and Satz 1 in [8] we have for any fixed admissible vertex tuple $P$ and corresponding polygon $\Gamma(P)$ :

\section{Proposition 2.2}

(i) $\psi(P, \cdot)$ is continuous on $T$.

(ii) $\quad f(P, \cdot):=\mathcal{D} \circ \psi(P, \cdot)$ is of class $C^{1}(T)$ and $\tilde{f}(P, \cdot):=\mathcal{D} \circ \tilde{\psi}(P, \cdot)$ even of class $C^{\omega}(T)$.

(iii) There holds $\tilde{f}(P, \tau)=f(P, \tau)$ if and only if $\tilde{\psi}(P, \tau)=\psi(P, \tau)$, which is again equivalent to $\tilde{\psi}(P, \tau) \in \mathcal{C}^{*}(\Gamma(P))$.

(iv) $\tilde{\psi}(P, \tau)$ and $\psi(P, \tau)$ are harmonic on $B \forall \tau \in T$.

(v) The restriction

$$
\left.\psi(P, \cdot)\right|_{K(f(P, \cdot))}: K(f(P, \cdot)) \stackrel{\cong}{\longrightarrow} \mathcal{M}(\Gamma(P))
$$

yields a homeomorphism between the compact set of critical points of $f(P, \cdot)$ and $\left(\mathcal{M}(\Gamma(P)),\|\cdot\|_{C^{0}(\bar{B})}\right)$, and a surface $\tilde{\psi}(P, \tau)$ is conformally parametrized on $B$, thus a minimal surface in $\tilde{\mathcal{U}}(P, \tau)$, if and only if $\tau$ is a critical point of $\tilde{f}(P, \cdot)$, i.e., $\tau \in K(\tilde{f}(P, \cdot))$.

(vi) Now we drop the fixed $P$ in the notation. For any $\bar{\tau} \in T$ and $k \in\{1, \ldots, N+3\}$ there exists some neighborhood $B_{\delta}\left(e^{i \bar{\tau}_{k}}\right) \times B_{\delta}^{N}(\bar{\tau})$ in $\mathbb{C} \times \mathbb{C}^{N}$ about $\left(e^{i \bar{\tau}_{k}}, \bar{\tau}\right)$ such that there holds the representation

$$
X_{w}(w, \tau)=\sum_{j=1}^{p_{k}} f_{j}^{k}(w, \tau)\left(w-e^{i \tau_{k}}\right)^{\rho_{j}^{k}}
$$


for $(w, \tau) \in\left(B_{\delta}\left(e^{i \bar{\tau}_{k}}\right) \cap B\right) \times\left(B_{\delta}^{N}(\bar{\tau}) \cap \mathbb{R}^{N}\right)$, where the functions $f_{j}^{k}$ are holomorphic on $B_{\delta}\left(e^{i \bar{\tau}_{k}}\right) \times B_{\delta}^{N}(\bar{\tau})$ about $\left(e^{i \bar{\tau}_{k}}, \bar{\tau}\right)$ and the exponents $\rho_{j}^{k}$ satisfy

$$
-1<\rho_{1}^{k}<\cdots<\rho_{p_{k}}^{k}=0, \quad p_{k} \in\{2,3\},
$$

and do not depend on $\tau \in B_{\delta}^{N}(\bar{\tau}) \cap \mathbb{R}^{N}$.

The last assertion about the independence of the exponents $\rho_{j}^{k}$ of $\tau \in B_{\delta}^{N}(\bar{\tau}) \cap \mathbb{R}^{N}$ follows immediately from [5], (2.20) and (3.28), as we point out now. We set $v_{k}:=\frac{P_{k+1}-P_{k}}{\left|P_{k+1}-P_{k}\right|}$ and consider as in (2.20) of [5] the reflections $S_{k}$ at the lines $\Gamma_{k}(P)-P_{k}=\operatorname{Span}\left(v_{k}\right)$ for $k \in\{1, \ldots, N+3\}$ (with $P_{N+4}:=P_{1}$ ), explicitly given by

$$
S_{k}(x):=-x+2\left\langle v_{k}, x\right\rangle v_{k}, \quad \forall x \in \mathbb{R}^{3} .
$$

The composed reflections $S_{k-1} \circ S_{k} \in \mathrm{SO}(3)$ are diagonalizable by conjugation with unitary matrizes and have eigenvalues on the $\mathbb{S}^{1}$. Now the $\rho_{j}^{k}$ appear in (3.28) of [5] as pairwise different (negative) angles of these eigenvalues, precisely:

$$
\operatorname{Spec}\left(S_{k-1} \circ S_{k}\right)=\left\{e^{-2 \pi i \rho_{j}^{k}}\right\}, \quad 1 \leq j \leq p_{k},
$$

ordered as in (6) with $p_{k} \in\{2,3\}$, which proves the claimed independence of the exponents $\rho_{j}^{k}$ of $\tau \in B_{\delta}^{N}(\bar{\tau}) \cap \mathbb{R}^{N}$. Moreover, we shall note that $S_{k-1} \circ S_{k} \neq \mathbf{1}$ and thus $p_{k}>1$ by our requirement that the vectors $P_{k-1}-P_{k}$ and $P_{k+1}-P_{k}$ have to be linearly independent. Moreover, we see that $p_{k}=2$ if and only if $\rho_{1}^{k}=-\frac{1}{2}$, i.e., if the spectrum of $S_{k-1} \circ S_{k}$ is $\{-1,-1,1\}$, which can arise if and only if the smaller angle $\beta_{k}$ between the vectors $P_{k-1}-P_{k}$ and $P_{k+1}-P_{k}$, the so-called interior angle of $\Gamma$ at $P_{k}$, is $\frac{\pi}{2}$. If in general $\beta_{k} \notin\left\{\frac{\pi}{2}, 0, \pi\right\}$, then the spectrum of $S_{k-1} \circ S_{k}$ is $\{\lambda, \bar{\lambda}, 1\}$ for some $\lambda \in \mathbb{S}^{1}$ with $\Im(\lambda) \neq 0$, i.e., $\rho_{1}^{k}+\rho_{2}^{k}=-1$. One can easily see that there holds either $-\rho_{1}^{k} \pi=\beta_{k}$ or $\left(\rho_{1}^{k}+1\right) \pi=\beta_{k}$, which is by $\rho_{1}^{k}+\rho_{2}^{k}=-1$ equivalent to the pair of possibilities $\left(\rho_{2}^{k}+1\right) \pi=\beta_{k}$ or $-\rho_{2}^{k} \pi=\beta_{k}$. Moreover, Heinz discovered in [7], Satz 2:

Proposition 2.3 If some vertex tuple $P^{*}$ satisfies the additional requirement that the interior angles at the vertices of $\Gamma\left(P^{*}\right)$ are different from $\frac{\pi}{2}$ then $X(\cdot, \cdot, \cdot)$ behaves analytically about a point $\left(w, P^{*}, \tau\right)$ in $B \times \mathbb{R}^{3 N+9} \times T$ for any fixed $w \in B$ and $\tau \in T$.

It should be mentioned here that the author could combine point (iv) of Proposition 2.2 with the Courant-Lebesgue Lemma, in order to prove in Chap. 4 of [13] that there holds for any admissible vertex tuple $P$ :

$$
\begin{aligned}
\tilde{\mathcal{M}}(\Gamma(P)) & :=\{\text { set of minimal surfaces on } B\} \cap \bigcup_{\tau \in T} \tilde{\mathcal{U}}(P, \tau) \cap H^{1,2}\left(B, \mathbb{R}^{3}\right) \\
& =\{X \in \operatorname{image}(\tilde{\psi}(P, \cdot)) \mid X \text { is also conformally parametrized on } B\} .
\end{aligned}
$$

Together with point (v) of Proposition 2.2 the author derived in Corollary 2.4 in [13] the following result, we shall make use of several times in the sequel:

Corollary 2.1 There holds $\mathcal{M}(\Gamma(P)) \subset \tilde{\mathcal{M}}(\Gamma(P))$ and also $K(f(P, \cdot)) \subset K(\tilde{f}(P, \cdot))$ for any admissible vertex tuple $P$. Moreover, $X(\cdot, P, \tau) \equiv \tilde{\psi}(P, \tau)$ coincides with $\psi(P, \tau)$ for any $\tau \in K(f(P, \cdot))$. 
Next, using this and point (v) of Proposition 2.2 we introduce the notation

$$
K_{s}(f(P, \cdot)):=(\psi(P, \cdot))^{-1}\left(\mathcal{M}_{s}(\Gamma(P))\right)
$$

for the set of critical points of $f(P, \cdot)$ that correspond to the immersed stable minimal surfaces in $\mathcal{C}^{*}(\Gamma(P))$. Furthermore, we will use the abbreviations $A^{(P, \tau)}:=-\triangle+2(K E)^{(P, \tau)}$ for the Schwarz operator and $J^{(P, \tau)}:=J^{X(\cdot, P, \tau)}$ for the quadratic form assigned to some minimal surface $X(\cdot, P, \tau)$, where $K^{(P, \tau)}$ denotes its Gauss curvature and $E^{(P, \tau)}:=1$ $\left.\frac{\partial}{\partial u} X(\cdot, P, \tau)\right|^{2}$.

As in [14] we will make use of the "Heinz formula" from [9], which states that for any fixed admissible $P \in \mathbb{R}^{3 N+9}$ and an arbitrary minimal surface $X(\cdot, P, \tau) \in \tilde{\mathcal{M}}(\Gamma(P))$ there holds the formula

$$
2 \kappa(P, \tau)+\operatorname{dim} \operatorname{Ker} A^{(P, \tau)}+\operatorname{rank}\left(D_{\tau}^{2} \tilde{f}(P, \tau)\right)=N
$$

where $\kappa(P, \tau):=\sum_{w \in B} m^{P, \tau}(w)+\frac{1}{2} \sum_{w \in \partial B} m^{P, \tau}(w)$ is the so-called total branch point order of $X(\cdot, P, \tau)$ (as defined in (10) in [14] for a fixed $P$ ), where $m^{P, \tau}(w)$ is defined as the power in the leading term of the Taylor expansion of $X(\cdot, P, \tau)$ about the point $w$, if $w \in \bar{B} \backslash\left\{e^{i \tau_{k}}\right\}_{k=1, \ldots, N+3}$, resp. as the integral contribution to the power in the leading term of (10), if the point $w$ coincides with $e^{i \tau_{k}}$ for some $k \in\{1, \ldots$, $N+3\}$.

In the proof of Theorem 1.2 we are going to combine the result of Theorem 1.1, the heart of its proof (by contradiction) in Sects. 6 and 7 in [14], certain special tools of it, e.g., Theorems 1.1 and 1.2 in [12] and the proof of Corollary 6 in [14], "Heinz' formula" (8) and the analytic dependence of $\tilde{f}(\cdot, \cdot)$ on both types of variables $P$ and $\tau$ by Satz 3 in Heinz' paper [7] with ideas of the proof of Tomi's quoted Theorem 1.3. As in Tomi's proof, we only have to examine the structure of the family of sets $K_{S}(f(P, \cdot))$, for $P$ varying in a small neighborhood in $\mathbb{R}^{3 N+9}$ of the corresponding vertex tuple $P^{*}$ of $\Gamma^{*}$, in a sufficiently small neighborhood of each (isolated) point of $K_{S}\left(f\left(P^{*}, \cdot\right)\right)$, and distinguish between the two cases in which such a point $\tau^{*}$ corresponds either to a strictly stable immersed minimal surface, i.e., in which the smallest eigenvalue $\lambda_{\min }\left(A^{\left(P^{*}, \tau^{*}\right)}\right)$ is positive, implying $\operatorname{rank}\left(D_{\tau}^{2}\left(\tilde{f}\left(P^{*}, \tau^{*}\right)\right)\right)=N$, or in which $\lambda_{\min }\left(A^{\left(P^{*}, \tau^{*}\right)}\right)=0$ and thus $\operatorname{rank}\left(D_{\tau}^{2}\left(\tilde{f}\left(P^{*}, \tau^{*}\right)\right)\right)=N-1$ by "Heinz' formula" (8) and Theorems 1.1 and 1.2 in [12]. In both cases we shall apply the implicit function theorem in its real analytic version. In the first case it yields the "stability" of immersed strictly stable minimal surfaces which span "jiggling" closed polygons in the common physical sense. Now a crucial idea due to Tomi was the use of Weierstrass' preparation theorem in a situation which corresponds to our second case. In our situation, the classical "finite-dimensional" version of Weierstrass' theorem can be applied to the composition $\Phi$ of a partial derivative of $\tilde{f}$, e.g., w.r.t. the coordinate $\tau_{N}$, with a certain $C^{\omega}$-graph in $\mathbb{R}^{3 N+9} \times T$ on which the remaining partial derivatives of $\tilde{f}$, i.e., w.r.t. $\tau_{1}, \ldots, \tau_{N-1}$, vanish. Hence, we obtain some Weierstrass polynomial as a factor of $\Phi$ whose degree will yield in fact an upper bound for the number of all minimal surfaces which span "jiggling" closed polygons $\Gamma$ close to $\Gamma^{*}$ and which are sufficiently close to some fixed stable minimal surface in $\mathcal{M}_{S}\left(\Gamma^{*}\right)$. It should be emphasized that the use of that analytic composition $\Phi$ (for some fixed $P$ ) already played a key role in the proof of Corollary 6 in [14]. 


\section{Proof of Theorem 1.1}

We fix some admissible vertex tuple $P$ and expand the holomorphic functions $f_{j}^{k}$ of Proposition 2.2 (vi) w.r.t. $w$ about the point $e^{i \tau_{k}}$ and obtain by (5) for any $k \in\{1, \ldots, N+3\}$ :

$$
X_{w}(w, \tau)=\sum_{j=1}^{p_{k}} \sum_{n=0}^{\infty} f_{j, n}^{k}(\tau)\left(w-e^{i \tau_{k}}\right)^{\rho_{j}^{k}+n}
$$

$\forall w \in B_{\delta}\left(e^{i \bar{\tau}_{k}}\right) \cap B$ and $\forall \tau \in B_{\delta}^{N}(\bar{\tau}) \cap \mathbb{R}^{N}$. Now we fix some $\bar{\tau} \in T$ and choose that pair $(j, n)$ for which $f_{j, n}^{k}(\bar{\tau}) \neq 0$ in $(9)$ and $\rho_{j}^{k}+n$ is minimal and term this pair $\left(j^{*}, m\right)$, i.e., we assign this pair to the point $\bar{\tau} \in T$. Since we know that either $\left(\rho_{j^{*}}^{k}+1\right) \pi$ or $-\rho_{j^{*}}^{k} \pi$ equals the smaller angle $\beta_{k} \neq 0, \pi$ between the linear independent vectors $P_{k-1}-P_{k}$ and $P_{k+1}-P_{k}$, we conclude due to $\rho_{p_{k}}^{k}=0$ that there has to hold $j^{*}<p_{k}$. Now using these terms, we derive formula (2.5) in [9] for some fixed admissible vertex tuple $P$ which we shall drop in the notation:

Corollary 3.1 For any fixed $\bar{\tau} \in T$ and $k \in\{1, \ldots, N+3\}$ there holds

$$
X_{w}(w, \bar{\tau})=f_{j^{*}, m}^{k}(\bar{\tau})\left(w-e^{i \bar{\tau}_{k}}\right)^{\rho_{j^{*}}^{k}+m}+O\left(\left|w-e^{i \bar{\tau}_{k}}\right|^{\rho_{j^{*}}^{k}+m+\epsilon}\right)
$$

for $B \ni w \rightarrow e^{i \bar{\tau}_{k}}$, where $\epsilon:=\rho_{j^{*}+1}^{k}-\rho_{j^{*}}^{k} \in(0,1)$.

Proof First, we note that $\epsilon$ is well defined by $j^{*}<p_{k}$. Now we split (9):

$$
X_{w}(w, \bar{\tau})=\sum_{j=1}^{p_{k}} f_{j, m}^{k}(\bar{\tau})\left(w-e^{i \bar{\tau}_{k}}\right)^{\rho_{j}^{k}+m}+F^{k}(w, \bar{\tau})
$$

where we set $F^{k}(w, \bar{\tau}):=\sum_{j=1}^{p_{k}} \sum_{n=m+1}^{\infty} f_{j, n}^{k}(\bar{\tau})\left(w-e^{i \bar{\tau}_{k}}\right)^{\rho_{j}^{k}+n}$ and shall show that there holds:

$$
\frac{\left|F^{k}(w, \bar{\tau})\right|}{\left|w-e^{i \bar{\tau}_{k}}\right|^{\rho_{j^{*}}^{k}+m+\epsilon}} \longrightarrow 0 \text { for } w \rightarrow e^{i \bar{\tau}_{k}}
$$

First, we have by $\rho_{j^{*}}^{k}+\epsilon=\rho_{j^{*}+1}^{k}$ :

$$
\frac{\left|F^{k}(w, \bar{\tau})\right|}{\left|w-e^{i \bar{\tau}_{k}}\right|^{\rho_{j^{*}}^{k}+m+\epsilon}} \leq \sum_{j=1}^{p_{k}} \sum_{n=1}^{\infty}\left|f_{j, n+m}^{k}(\bar{\tau})\right|\left|w-e^{i \bar{\tau}_{k}}\right|^{n+\rho_{j}^{k}-\rho_{j^{*}+1}^{k}}
$$

$\forall w \in B_{\delta}\left(e^{i \bar{\tau}_{k}}\right) \cap B$. Now applying Cauchy's inequalities to the Taylor coefficients $f_{j, n+m}^{k}(\bar{\tau})$, we achieve:

$$
\left|f_{j, n+m}^{k}(\bar{\tau})\right| \leq \frac{c(\hat{\delta})}{\hat{\delta}^{n+m}} \quad \forall n \in \mathbb{N},
$$

for any $j \in\left\{1, \ldots, p_{k}\right\}$ and some arbitrarily fixed $\hat{\delta} \in(0, \delta)$ with

$$
c(\hat{\delta}):=c(\hat{\delta}, \bar{\tau}, k):=\sqrt{3} \max _{j \in\left\{1, \ldots, p_{k}\right\}} \max _{\partial B_{\hat{\delta}}\left(e^{i \bar{\tau}}\right)}\left|f_{j}^{k}(\cdot, \bar{\tau})\right| .
$$


Hence, using $p_{k} \leq 3, \rho_{j}^{k} \geq \rho_{1}^{k}$ and $\sum_{n=0}^{\infty} 2^{-n}=2$ we obtain:

$$
\begin{aligned}
& \frac{\left|F^{k}(w, \bar{\tau})\right|}{\left|w-e^{i \bar{\tau}_{k}}\right|^{\rho_{j^{*}}^{k}+m+\epsilon}} \leq 3 \frac{c(\hat{\delta})}{\hat{\delta}^{m+1}}\left|w-e^{i \bar{\tau}_{k}}\right|^{1+\rho_{1}^{k}-\rho_{j^{*}+1}^{k}} \sum_{n=1}^{\infty} \frac{\left|w-e^{i \bar{\tau}_{k}}\right|^{n-1}}{\hat{\delta}^{n-1}} \\
& \leq 3 \frac{c(\hat{\delta})}{\hat{\delta}^{m+1}}\left|w-e^{i \bar{\tau}_{k}}\right|^{1+\rho_{1}^{k}-\rho_{j^{*}+1}^{k}} \sum_{n=0}^{\infty} 2^{-n}=6 \frac{c(\hat{\delta})}{\hat{\delta}^{m+1}}\left|w-e^{i \bar{\tau}_{k}}\right|^{1+\rho_{1}^{k}-\rho_{j^{*}+1}^{k}}
\end{aligned}
$$

$\forall w \in B_{\frac{\delta}{2}}\left(e^{i \bar{\tau}_{k}}\right) \cap B$. Thus taking $1+\rho_{1}^{k}-\rho_{j^{*}+1}^{k}>0$ into account we achieve (12), which proves the corollary.

Now we fix some admissible vertex tuple $P$ (which we will drop in the notation) such that $\Gamma(P)$ meets the requirement of Theorem 1.1 and prove the following generalization of assertion (b) in the proof of Theorem 8 in [14], where the polygon was required to be contained in the boundary of a convex compact set and not in a plane. In fact these requirements imply the one imposed on $\Gamma$ in Theorem 1.1 by Theorem 4 in [14] resp. Theorem 4.1 in [13].

Theorem 3.1 Suppose $\bar{\tau} \in K(\tilde{f})$ has the properties rank $D^{2}(\tilde{f})(\bar{\tau})=N-1$ and $\kappa(\bar{\tau})=0$. Then there exists some $\delta>0$ such that $\kappa \equiv 0$ on $B_{\delta}(\bar{\tau}) \cap K(\tilde{f})$.

Proof By rank $D^{2}(\tilde{f})(\bar{\tau})=N-1$, we can conclude that there exists some $\delta>0$ such that $\operatorname{rank} D^{2}(\tilde{f}) \geq N-1$ on $B_{\delta}(\bar{\tau})$. Thus by Heinz' formula (8) we obtain $2 \kappa(\tau) \leq 1$ $\forall \tau \in B_{\delta}(\bar{\tau}) \cap K(\tilde{f})$. Hence, $X(\cdot, \tau)$ can have at most one simple boundary branch point $w^{*}(\tau) \in \partial B$ for those $\tau$. Moreover, we claim that there would have to hold $X\left(w^{*}, \tau\right) \notin \Gamma$. For, if there would hold $X\left(w^{*}, \tau\right) \in \Gamma$ we could conclude that $X(\cdot, \tau) \in \mathcal{M}(\Gamma)$, i.e., that $X(\cdot, \tau)$ would have to be bounded by $\Gamma$, as otherwise $X(\cdot, \tau)$ would have to possess another boundary branch point by Lemma 4 in [14]. But, any surface in $\mathcal{M}(\Gamma)$ is required to be free of boundary branch points, which thus proves our claim. This especially shows that the points $e^{i \tau_{l}}$ for $l \in\{1, \ldots, N+3\}$ cannot be branch points of $X(\cdot, \tau)$ whenever $\tau \in B_{\delta}(\bar{\tau}) \cap K(\tilde{f})$. Now we suppose that there would exist some sequence $\left\{\tau^{n}\right\} \subset B_{\delta}(\bar{\tau}) \cap K(\tilde{f})$ with $\tau^{n} \rightarrow \bar{\tau}$ such that $X\left(\cdot, \tau^{n}\right)$ has exactly one simple boundary branch point $w^{n} \in \partial B \backslash\left\{e^{i \tau_{l}^{n}}\right\}_{l=1, \ldots, N+3}$ for each $n \in \mathbb{N}$. One can easily show that there exists some $k \in\{1, \ldots, N+3\}$ and some subsequence $\left\{w^{n_{j}}\right\}$ such that for each $j$ the point $w^{n_{j}}$ is contained in the open $\operatorname{arc}\left\{e^{i \theta} \mid \theta \in\left(\tau_{k}^{n_{j}}, \tau_{k+1}^{n_{j}}\right)\right\}$. For simplicity we rename the sequence $\left\{n_{j}\right\}$ into $\{n\}$ again. Now for each fixed $n$ there are again two possibilities: either (a) $X\left(w^{n}, \tau^{n}\right) \in\left\{P_{k}+t\left(P_{k+1}-P_{k}\right) \mid t<0\right\}$ or (b) $X\left(w^{n}, \tau^{n}\right) \in\left\{P_{k}+t\left(P_{k+1}-P_{k}\right) \mid t>1\right\}$. Since there has to exist some further subsequence $\left\{n_{j}\right\}$ such that there holds either Case (a) or Case (b) for every $j$ we may assume that the first case would hold for each $n$ without loss of generality. This case means that the boundary values $X\left(e^{i \theta}, \tau^{n}\right)$ of the surfaces $X\left(\cdot, \tau^{n}\right)$ "overshoot" onto $\Gamma_{k} \backslash \Gamma$ immediately after they have passed the vertex $P_{k}$, i.e., immediately after $\theta$ has exceeded the value $\tau_{k}^{n}$. Now by (11) and $\rho_{p_{l}}^{l}=0$ we have for any fixed $\tau \in B_{\delta}(\bar{\tau}) \cap K(\tilde{f})$ and each $l \in\{1, \ldots, N+3\}$ :

$$
X_{w}(w, \tau)=f_{1,0}^{l}(\tau)\left(w-e^{i \tau_{l}}\right)^{\mu_{1}^{l}}+f_{2,0}^{l}(\tau)\left(w-e^{i \tau_{l}}\right)^{\mu_{2}^{l}}+f_{3,0}^{l}(\tau)+F^{l}(w, \tau),
$$

$\forall w \in B_{\delta}\left(e^{i \bar{\tau}_{l}}\right) \cap B$, where $-1<\mu_{1}^{l} \leq \mu_{2}^{l}<0$ with $\mu_{1}^{l}+\mu_{2}^{l}=-1$. Now we claim that it is impossible that $f_{1,0}^{l}(\tau)=f_{2,0}^{l}(\tau)=0$. Suppose the claim were wrong. Then, either we would have also $f_{3,0}^{l}(\tau)=0$, but then the point $e^{i \tau_{l}}$ would be a branch point of $X(\cdot, \tau)$, which we ruled out above, or there would hold $f_{3,0}^{l}(\tau) \neq 0$, but then the surface $X(\cdot, \tau)$ 
would form the angle $\pi$ in the corner $P_{l}$, i.e., then $P_{l}$ would not be a vertex of $\Gamma$, see (2). Hence, there are only two possibilities:

(i) There holds $f_{1,0}^{l}(\tau) \neq 0$, (ii) $f_{1,0}^{l}(\tau)=0$ but $f_{2,0}^{l}(\tau) \neq 0$. There holds Case (i) if and only if $X(\cdot, \tau)$ forms the angle $\pi\left(1+\mu_{1}^{l}\right)$ at the corner $P_{l}$ and Case (ii) if and only if $X(\cdot, \tau)$ forms the angle $\pi\left(1+\mu_{2}^{l}\right)$ at the corner $P_{l}$. We define the so-called geodesic angle $\delta_{l}(\tau)$ to be $-\pi \mu_{1}^{l}=\pi\left(1+\mu_{2}^{l}\right)$ in the first case and $-\pi \mu_{2}^{l}=\pi\left(1+\mu_{1}^{l}\right)$ in the second case. Now, the minimal surfaces $X\left(\cdot, \tau^{n}\right)$ all have to form the same angle at each corner $P_{l}$, since their boundary values all "overshoot" onto $\Gamma_{k} \backslash \Gamma$ immediately after those have passed the vertex $P_{k}$, meaning that they form the angle $\arccos \left(\frac{\left\langle P_{k-1}-P_{k}, P_{k}-P_{k+1}\right\rangle}{\left|P_{k-1}-P_{k}\right|\left|P_{k}-P_{k+1}\right|}\right)$ in $P_{k}$ for each $n$, and since for $l \neq k$ each $X\left(\cdot, \tau^{n}\right)$ maps some neighborhood of $e^{i \tau_{l}^{n}}$ in $\mathbb{S}^{1}$ monotonically and continuously onto some neighborhood of the vertex $P_{l}$ in $\Gamma$ (thus each $X\left(\cdot, \tau^{n}\right)$ meets the usual "Plateau boundary condition" about $e^{i \tau_{l}^{n}}$ for $\left.l \neq k\right)$, meaning that every $X\left(\cdot, \tau^{n}\right)$ forms the angle arccos $\left(\frac{\left\langle P_{l-1}-P_{l}, P_{l+1}-P_{l}\right\rangle}{\left|P_{l-1}-P_{l}\right|\left|P_{l+1}-P_{l}\right|}\right)$ in each $P_{l}$, for $l \neq k$. In other terms, the minimal surfaces $X\left(\cdot, \tau^{n}\right)$ have to meet simultaneously for every $n$ either only the above Case (i) or only the Case (ii) for each $l$ respectively, implying that $\delta_{l}\left(\tau^{n}\right) \equiv \delta_{l} \forall n \in \mathbb{N}$ and for each $l$. Now $\tau^{n} \longrightarrow \bar{\tau}$ implies that $K E\left(w, \tau^{n}\right) \longrightarrow K E(w, \bar{\tau})$ pointwise $\forall w \in B$ since $X\left(\cdot, \tau^{n}\right)$ and $X(\cdot, \bar{\tau})$ are free of interior branch points. Thus also noting that these functions are integrable (on account of estimate (26) in [14]) and non-positive on $B$ we infer from Fatou's Lemma that

$$
\int_{B}-K E(w, \bar{\tau}) d w \leq \liminf _{n \rightarrow \infty} \int_{B}-K E\left(w, \tau^{n}\right) d w .
$$

Now combining this with Sauvigny's version of Gauss-Bonnet's Theorem for minimal surfaces in $\tilde{\mathcal{M}}(\Gamma)$ (see [17]) applied to each $X\left(\cdot, \tau^{n}\right)$, i.e., with

$$
\int_{B} K E\left(w, \tau^{n}\right) d w+\sum_{l=1}^{N+3} \delta_{l}\left(\tau^{n}\right)=2 \pi\left(1+\kappa\left(\tau^{n}\right)\right)=3 \pi,
$$

and with the constancy of the geodesic angles $\delta_{l}\left(\tau^{n}\right)=: \delta_{l} \quad \forall n \in \mathbb{N}$ and for each $l \in$ $\{1, \ldots, N+3\}$ we arrive at

$$
-\int_{B} K E(w, \bar{\tau}) d w \leq \sum_{l=1}^{N+3} \delta_{l}-3 \pi .
$$

On the other hand Sauvigny's Gauss-Bonnet Theorem applied to the immersion $X(\cdot, \bar{\tau})$ yields

$$
\int_{B} K E(w, \bar{\tau}) d w+\sum_{l=1}^{N+3} \delta_{l}(\bar{\tau})=2 \pi(1+\kappa(\bar{\tau}))=2 \pi .
$$

Now, we have assumed that the boundary values of the surfaces $X\left(\cdot, \tau^{n}\right)$ "overshoot" onto $\Gamma_{k} \backslash \Gamma$ immediately after they have passed the vertex $P_{k}$. Hence, the surfaces $X\left(\cdot, \tau^{n}\right)$ map into the so-called exterior angle of $\Gamma$ at $P_{k}$. Moreover we have assumed that $X(\cdot, \bar{\tau})$ is free of branch points on $\bar{B}$, and thus, bounded by $\Gamma$. Hence, $X(\cdot, \bar{\tau})$ maps into the interior angle at each vertex $P_{l}$ of $\Gamma$. This yields $\delta_{l}(\bar{\tau})=\delta_{l}\left(\tau^{n}\right) \equiv \delta_{l}$ for each $l \neq k$ and $\delta_{k}(\bar{\tau})=\pi-\delta_{k}\left(\tau^{n}\right) \equiv \pi-\delta_{k}$ for $l=k, \forall n \in \mathbb{N}$. Hence, we can compute now

$$
\sum_{l=1}^{N+3}\left(\delta_{l}(\bar{\tau})-\delta_{l}\left(\tau^{n}\right)\right)=\delta_{k}(\bar{\tau})-\delta_{k}\left(\tau^{n}\right)=\pi-2 \delta_{k} .
$$


Combining this with (14), we arrive at

$$
\begin{aligned}
-\int_{B} K E(w, \bar{\tau}) d w & =\sum_{l=1}^{N+3} \delta_{l}\left(\tau^{n}\right)+\pi-2 \delta_{k}-2 \pi=\sum_{l=1}^{N+3} \delta_{l}-\left(\pi+2 \delta_{k}\right) \\
& >\sum_{l=1}^{N+3} \delta_{l}-3 \pi,
\end{aligned}
$$

where we used that $\delta_{k}<\pi$ due to $\mu_{2}^{k} \geq \mu_{1}^{k}>-1$. Since this contradicts (13), we have proved the theorem.

Having proved this one achieves that for a point $\bar{\tau}$ as in Theorem 3.1 there exists some $\delta>0$ such that $B_{\delta}(\bar{\tau}) \cap K(\tilde{f})=B_{\delta}(\bar{\tau}) \cap K(f)$, thus especially the assertion of part (c) of the proof of Theorem 8 in [14]. Therefore, one can apply estimate (45) in [14] in order to prove the convergence in (46) in [14], and thus the entire rest of the proof of the finiteness theorem in [14] can be copied in order to prove its generalization, Theorem 1.1.

\section{Proof of Theorem 1.2}

Now we fix some polygon $\Gamma^{*} \subset \mathbb{R}^{3}$ as in Theorem 1.2 with vertex tuple $P^{*}$. Following Tomi's ideas of the proof of Lemma 4 in [19], we shall prove analogously:

Lemma 4.1 Let $U \subset \subset T$ be some arbitrary open neighborhood of the finite (nonvoid) set $K_{S}\left(f\left(P^{*}, \cdot\right)\right)$. Then there exists some neighborhood $O$ of $P^{*}$ in $\mathbb{R}^{3 N+9}$ such that there holds

$$
K_{s}(f(P, \cdot)) \subset U \quad \forall P \in O .
$$

Proof We suppose the statement was wrong. Thus there would have to exist some sequence $\left\{P^{n}\right\}$ in $\mathbb{R}^{3 N+9}$ with

$$
P^{n} \longrightarrow P^{*}, \text { for } n \rightarrow \infty,
$$

and according points $\tau^{n} \in K_{S}\left(f\left(P^{n}, \cdot\right)\right) \cap U^{c} \forall n \in \mathbb{N}$. Clearly there exists some converging subsequence $\left\{\tau^{n_{j}}\right\}$, which we rename $\left\{\tau^{n}\right\}$ again, and we claim the limit point $\tau^{*}$ to be contained in $T$, i.e.:

$$
\tau^{n} \longrightarrow \tau^{*} \in T \cap U^{c}
$$

For let us assume that there would hold $\operatorname{dist}\left(\tau^{n}, \partial T\right) \longrightarrow 0$. By the definition of $T$ this assumption implies the existence of some subsequence $\left\{\tau^{n_{k}}\right\}$ and some consecutive indices $l_{1}, l_{2}=l_{1}+1$ in $\{1, \ldots, N+3\}$ (where $l_{1}:=N+3$ if $l_{2}=1$ or $l_{2}:=1$ if $l_{1}=N+3$ ) and some angle $\varphi \in[0, \pi] /(0 \sim 2 \pi)$ with

$$
\tau_{l_{1}}^{n_{k}} \longrightarrow \varphi \longleftarrow \tau_{l_{2}}^{n_{k}}
$$

Now, we consider the null sequence $\delta_{k}:=\frac{1}{8 k}+\max _{j=1,2}\left\{\left|e^{i \tau_{l_{j}{ }_{k}}}-e^{i \varphi}\right|\right\}$ which we may assume to be contained in $(0,1)$ for any $k \in \mathbb{N}$. By the Courant-Lebesgue Lemma, applied to the surfaces $X\left(\cdot, P^{n_{k}}, \tau^{n_{k}}\right)$, there exists some $\rho_{k} \in\left(\delta_{k}, \sqrt{\delta_{k}}\right)$ such that there holds

$$
\left|X\left(w_{1}^{k}, P^{n_{k}}, \tau^{n_{k}}\right)-X\left(w_{2}^{k}, P^{n_{k}}, \tau^{n_{k}}\right)\right| \leq \sqrt{\frac{4 \pi \mathcal{D}\left(X\left(\cdot, P^{n_{k}}, \tau^{n_{k}}\right)\right)}{\log \frac{1}{\delta_{k}}}}
$$


in the points $\left\{w_{1}^{k}, w_{2}^{k}\right\}:=\partial B_{\rho_{k}}\left(e^{i \varphi}\right) \cap \partial B$ and $\forall k \in \mathbb{N}$. Moreover, by (15) the corresponding polygons $\Gamma^{n}:=\Gamma\left(P^{n}\right)$ satisfy a uniform chord-arc condition, i.e., there exists some constant $C$ such that there holds for any fixed $n \in \mathbb{N}$ and any pair of points $Q_{1}, Q_{2} \in \Gamma^{n}$ :

$$
\mathcal{L}\left(\left.\Gamma^{n}\right|_{\left(Q_{1}, Q_{2}\right)}\right) \leq C\left|Q_{1}-Q_{2}\right|,
$$

where $\mathcal{L}\left(\left.\Gamma^{n}\right|_{\left(Q_{1}, Q_{2}\right)}\right)$ denotes the length of the shorter subarc $\left.\Gamma^{n}\right|_{\left(Q_{1}, Q_{2}\right)}$ on $\Gamma^{n}$ connecting $Q_{1}$ and $Q_{2}$. Furthermore we know that there exists some constant $L$ such that $\mathcal{L}\left(\Gamma^{n}\right) \leq L$ $\forall n \in \mathbb{N}$ again due to (15). Finally, since the $\tau^{n}$ are assumed to be critical points of $f\left(P^{n}, \cdot\right)$ implying that $X\left(\cdot, P^{n}, \tau^{n}\right) \equiv \tilde{\psi}\left(P^{n}, \tau^{n}\right)$ coincide with $\psi\left(P^{n}, \tau^{n}\right)$ by Corollary 2.1 and thus are elements of $\mathcal{M}\left(\Gamma^{n}\right)$ the isoperimetric inequality yields:

$$
\mathcal{D}\left(X\left(\cdot, P^{n}, \tau^{n}\right)\right)=\mathcal{A}\left(X\left(\cdot, P^{n}, \tau^{n}\right)\right) \leq \frac{1}{4 \pi} L^{2} \quad \forall n \in \mathbb{N} .
$$

Now combining this with (17) and (18), applied to $Q_{j}^{k}:=X\left(w_{j}^{k}, P^{n_{k}}, \tau^{n_{k}}\right)$ for $j=1,2$, we arrive at:

$$
\mathcal{L}\left(\left.\Gamma^{n_{k}}\right|_{\left(Q_{1}^{k}, Q_{2}^{k}\right)}\right) \leq \frac{C L}{\sqrt{\log \frac{1}{\delta_{k}}}} \longrightarrow 0 \text { for } k \rightarrow \infty .
$$

Together with (15) this shows in particular that $\left.\Gamma^{n_{k}}\right|_{\left(Q_{1}^{k}, Q_{2}^{k}\right)}$ can contain at most one of the points $\left\{P_{N+l}^{n_{k}}\right\}_{l=1,2,3}$ of the respective three-point condition for sufficiently large $k$. Moreover, $B_{\rho_{k}}\left(e^{i \varphi}\right) \cap \partial B$ can contain at most one of the three-points $\left\{e^{i \pi}, e^{i \frac{3}{2} \pi}, 1\right\}$ of the three-point condition for sufficiently large $k$ on account of $\rho_{k}<\sqrt{\delta_{k}} \rightarrow 0$. Together with the weak monotonicity and the three-point condition imposed on the boundary values $\left.X\left(\cdot, P^{n_{k}}, \tau^{n_{k}}\right)\right|_{\partial B}$ this guarantees that

$$
\operatorname{trace}\left(\left.X\left(\cdot, P^{n_{k}}, \tau^{n_{k}}\right)\right|_{B_{\rho_{k}}\left(e^{i \varphi}\right) \cap \partial B}\right)=\left.\Gamma^{n_{k}}\right|_{\left(Q_{1}^{k}, Q_{2}^{k}\right)} \quad \forall k>K,
$$

for some large $K \in \mathbb{N}$. Moreover, there holds $e^{i \tau_{l_{j}}^{n_{k}}} \in B_{\rho_{k}}\left(e^{i \varphi}\right) \cap \partial B$, for any $k$ and $j=1,2$, by $\rho_{k}>\delta_{k}$ and the definition of $\delta_{k}$. Thus we can infer from (3) and (21) that $P_{l_{j}}^{n_{k}}=$ $\left.X\left(e^{i \tau_{l_{j}}^{n_{k}}}, P^{n_{k}}, \tau^{n_{k}}\right) \in \Gamma^{n_{k}}\right|_{\left(Q_{1}^{k}, Q_{2}^{k}\right)}$, for $j=1,2$ and $k>K$, and thus together with (20):

$$
\left|P_{l_{1}}^{n_{k}}-P_{l_{2}}^{n_{k}}\right| \leq \mathcal{L}\left(\left.\Gamma^{n_{k}}\right|_{\left(Q_{1}^{k}, Q_{2}^{k}\right)}\right) \longrightarrow 0 \text { for } k \rightarrow \infty,
$$

which contradicts $\left|P_{l_{1}}^{n_{k}}-P_{l_{2}}^{n_{k}}\right| \rightarrow\left|P_{l_{1}}^{*}-P_{l_{2}}^{*}\right|>0$ by (15). Thus, we proved in fact (16). Now, we know by Proposition 2.3 that $X(\cdot, \cdot, \cdot)$ behaves analytically about a point $\left(w, P^{*}, \tau^{*}\right)$ in $B \times \mathbb{R}^{3 N+9} \times T$ for any fixed $w \in B$, where we use the additional requirement on $P^{*}$ that the interior angles at the vertices of $\Gamma^{*} \equiv \Gamma\left(P^{*}\right)$ are different from $\frac{\pi}{2}$ (Requirement $(\mathrm{H})$ in [7]). Thus we conclude in particular by (15) and (16) that

$$
X\left(w, P^{n_{k}}, \tau^{n_{k}}\right) \longrightarrow X\left(w, P^{*}, \tau^{*}\right) \text { for } k \rightarrow \infty,
$$

pointwise for any $w \in B$. Moreover, (15) implies the Frechet convergence of $\left\{\Gamma^{n}\right\}$ to $\Gamma^{*}$. Thus together with $X\left(\cdot, P^{n_{k}}, \tau^{n_{k}}\right) \in \mathcal{M}\left(\Gamma^{n_{k}}\right)$ and (19) we see that all requirements of a compactness result for boundary values due to Nitsche (see [16], p. 208) are fulfilled which implies the existence of some further subsequence of $\left\{X\left(\cdot, P^{n_{k}}, \tau^{n_{k}}\right)\right\}$ (to be renamed again) that satisfies:

$$
\left.X\left(\cdot, P^{n_{k}}, \tau^{n_{k}}\right)\right|_{\partial B} \longrightarrow \gamma \quad \text { in } C^{0}\left(\partial B, \mathbb{R}^{3}\right)
$$


for some continuous, weakly monotonic map $\gamma: \partial B \longrightarrow \Gamma^{*}$ onto $\Gamma^{*}$ satisfying the appropriate three point condition $\gamma\left(e^{i \tau_{N+l}}\right)=P_{N+l}^{*}$, for $l=1,2,3$. Now let $\bar{X}$ denote the unique harmonic extension of $\gamma$ onto $\bar{B}$, thus being contained in $\mathcal{C}^{*}\left(\Gamma^{*}\right)$. Due to (23) we infer from the maximum principle for harmonic functions and Cauchy's estimates:

$$
X\left(\cdot, P^{n_{k}}, \tau^{n_{k}}\right) \longrightarrow \bar{X} \text { in } C^{0}\left(\bar{B}, \mathbb{R}^{3}\right) \text { and } C_{l o c}^{1}\left(B, \mathbb{R}^{3}\right)
$$

for $k \rightarrow \infty$. In combination with (22), we obtain especially that $\bar{X}$ and $X\left(\cdot, P^{*}, \tau^{*}\right)$ coincide on $B$ and thus on $\bar{B}$ and that they are contained in $\mathcal{M}\left(\Gamma^{*}\right)$ on account of the properties of $\gamma$ and (24). Now, since the minimal surfaces $X\left(\cdot, P^{n_{k}}, \tau^{n_{k}}\right)$ do not have any branch points on $\bar{B}$ and are stable we can apply Theorem 1 in [18] which yields that the limit surface $\bar{X}$ is free of interior branch points again. Moreover, since $\bar{X}$ is bounded by the polygon $\Gamma^{*}$ which we require to bound only minimal surfaces without boundary branch points, $\bar{X}$ is an immersed minimal surface. Moreover, (24) yields by the proof of Theorem 3 in [14] that $\bar{X}$ is also stable again. Hence, all together we infer that $\bar{X}=X\left(\cdot, P^{*}, \tau^{*}\right) \in \mathcal{M}_{S}\left(\Gamma^{*}\right)$. By point (iii) of Proposition 2.2 we obtain especially that $\bar{X} \equiv \tilde{\psi}\left(P^{*}, \tau^{*}\right)=\psi\left(P^{*}, \tau^{*}\right)$. Thus, we arrive at $\tau^{*}=\psi\left(P^{*}, \cdot\right)^{-1}(\bar{X}) \in K_{S}\left(f\left(P^{*}, \cdot\right)\right)$, in contradiction to $\tau^{*} \notin U$ by (16).

Now we can finally derive the proof of Theorem 1.2:

Let $P^{*}$ be the vertex tuple corresponding to the polygon $\Gamma^{*}$. By Theorem 1.1, Proposition 2.1 and (7) we know that $K_{S}\left(f\left(P^{*}, \cdot\right)\right)$ is a nonvoid and finite subset of $T$. Now we choose one of its points $\tau^{*}$ arbitrarily and recall that $X\left(\cdot, P^{*}, \tau^{*}\right) \equiv \tilde{\psi}\left(P^{*}, \tau^{*}\right)$ coincides with $\psi\left(P^{*}, \tau^{*}\right)$ by Corollary 2.1 and is thus an element of $\mathcal{M}_{S}\left(\Gamma^{*}\right)$. Therefore, we have $J^{\left(P^{*}, \tau^{*}\right)} \geq 0$ on $C_{c}^{\infty}(B)$ and thus on $\stackrel{H}{H}^{1,2}(B)$ by Lemma 2 in [14], which implies that there can arise only the following two cases for the smallest eigenvalue $\lambda_{\min }\left(A^{\left(P^{*}, \tau^{*}\right)}\right)$ of the Schwarz operator $A^{\left(P^{*}, \tau^{*}\right)}$ on account of Theorem 1.1 in [12]:

$$
\text { Case 1: } \lambda_{\min }\left(A^{\left(P^{*}, \tau^{*}\right)}\right)>0 \text { or Case 2: } \lambda_{\min }\left(A^{\left(P^{*}, \tau^{*}\right)}\right)=0 .
$$

In Case 1, we infer that $\operatorname{kernel}\left(A^{\left(P^{*}, \tau^{*}\right)}\right)=\{0\}$. Thus in combination with the fact that $X\left(\cdot, P^{*}, \tau^{*}\right)$ is an immersed minimal surface, we can immediately derive from formula (8) that

$$
\operatorname{rank} D_{\tau}^{2}\left(\tilde{f}\left(P^{*}, \tau^{*}\right)\right)=N
$$

Since $\tilde{f}$ is real analytic, thus smooth in particular, about the point $\left(P^{*}, \tau^{*}\right)$ by Satz 3 in [7] there exists some neighborhood $O^{*} \times U^{*}$ of $\left(P^{*}, \tau^{*}\right)$ in $\mathbb{R}^{3 N+9} \times T$ such that there holds:

$$
\operatorname{det} D_{\tau}\left(\nabla_{\tau} \tilde{f}(P, \tau)\right) \neq 0 \quad \forall(P, \tau) \in O^{*} \times U^{*} .
$$

Also noting that the point $\left(P^{*}, \tau^{*}\right)$ solves the $N$ equations $\nabla_{\tau} \tilde{f}(P, \tau)=0$ the implicit function theorem in its real analytic version (see, e.g., [4], p. 268) applied to $\nabla_{\tau} \tilde{f} \in C^{\omega}\left(O^{*} \times\right.$ $U^{*}, \mathbb{R}^{N}$ ) yields:

There exist neighborhoods $U_{1}^{*} \subset U^{*}$ of $\tau^{*}$ and $O_{1}^{*} \subset O^{*}$ of $P^{*}$, where $O_{1}^{*}$ has to be chosen sufficiently small depending on the choice of $U_{1}^{*}$, and some function $F \in C^{\omega}\left(O_{1}^{*}, U_{1}^{*}\right)$, such that there holds $F\left(P^{*}\right)=\tau^{*}$ and

$$
\left\{(P, \tau) \in O_{1}^{*} \times U_{1}^{*} \mid \nabla_{\tau} \tilde{f}(P, \tau)=0\right\}=\left\{(P, F(P)) \mid P \in O_{1}^{*}\right\}=\operatorname{graph}(F) .
$$

Hence, for any $P \in O_{1}^{*}$ there holds:

$$
K(\tilde{f}(P, \cdot)) \cap U_{1}^{*}=\{F(P)\}
$$


and thus by $K_{S}(f(P, \cdot)) \subset K(\tilde{f}(P, \cdot))$ on account of Corollary 2.1:

$$
\sharp\left(K_{S}(f(P, \cdot)) \cap U_{1}^{*}\right) \leq 1 .
$$

In Case 2 we infer from Theorem 1.2 in [12] that

$$
\operatorname{dim} \operatorname{Ker}\left(A^{\left(P^{*}, \tau^{*}\right)}\right)=\operatorname{dim} \operatorname{ER}_{\lambda_{\min }=0}\left(A^{\left(P^{*}, \tau^{*}\right)}\right)=1,
$$

which implies again by formula (8) that

$$
\operatorname{rank} D_{\tau}^{2}\left(\tilde{f}\left(P^{*}, \tau^{*}\right)\right)=N-1 .
$$

Thus, after some eventual permutation of the coordinates $\tau_{1}, \ldots, \tau_{N}$ in $T$ we may assume that there exists some neighborhood $O^{*} \times U^{*}$ of the point $\left(P^{*}, \tau^{*}\right)$ in $\mathbb{R}^{3 N+9} \times T$ such that there holds:

$$
\operatorname{det} D_{\hat{\tau}}\left(\nabla_{\hat{\tau}} \tilde{f}\left(P,\left(\hat{\tau}, \tau_{N}\right)\right)\right) \neq 0 \quad \forall\left(P,\left(\hat{\tau}, \tau_{N}\right)\right) \in O^{*} \times U^{*},
$$

where we set $\hat{\tau}:=\left(\tau_{1}, \ldots, \tau_{N-1}\right)$. Now let $\hat{U} \subset \mathbb{R}^{N-1}$ and $I \subset \mathbb{R}$ be neighborhoods of $\hat{\tau}^{*}$ and $\tau_{N}^{*}$, respectively, such that $\hat{U} \times I \subset U^{*}$. Also noting that $\left(P^{*}, \hat{\tau}^{*}, \tau_{N}^{*}\right)$ is a solution of the $N-1$ equations $\nabla_{\hat{\tau}} \tilde{f}\left(P, \hat{\tau}, \tau_{N}\right)=0$ the implicit function theorem in its real analytic version applied to $\nabla_{\hat{\tau}} \tilde{f} \in C^{\omega}\left(O^{*} \times \hat{U} \times I, \mathbb{R}^{N-1}\right)$ (by Satz 3 in [7]) yields:

There exist neighborhoods $\hat{U}_{1} \subset \hat{U}$ of $\hat{\tau}, O_{1}^{*} \subset O^{*}$ of $P^{*}$ and $I_{1} \subset I$ of $\tau_{N}^{*}$, where $O_{1}^{*}$ and $I_{1}$ have to be chosen sufficiently small depending on the choice of $\hat{U}_{1}$, and some function $F \in C^{\omega}\left(O_{1}^{*} \times I_{1}, \hat{U}_{1}\right)$ such that there holds $F\left(P^{*}, \tau_{N}^{*}\right)=\hat{\tau}^{*}$ and

$$
\begin{aligned}
& \left\{\left(P, \hat{\tau}, \tau_{N}\right) \in O_{1}^{*} \times \hat{U}_{1} \times I_{1} \mid \nabla_{\hat{\tau}} \tilde{f}\left(P, \hat{\tau}, \tau_{N}\right)=0\right\} \\
& \quad=\left\{\left(P, F\left(P, \tau_{N}\right), \tau_{N}\right) \mid\left(P, \tau_{N}\right) \in O_{1}^{*} \times I_{1}\right\}=\operatorname{graph}(F) .
\end{aligned}
$$

Now let some $P \in O_{1}^{*}$ be arbitrarily chosen, then a point $\left(F\left(P, \tau_{N}\right), \tau_{N}\right) \in \hat{U}_{1} \times I_{1}$ is contained in $K(\tilde{f}(P, \cdot)) \cap\left(\hat{U}_{1} \times I_{1}\right)$ if and only if it satisfies $\nabla_{\tau} \tilde{f}\left(P, F\left(P, \tau_{N}\right), \tau_{N}\right)=0$, which is by (30) equivalent to

$$
\Phi\left(P, \tau_{N}\right):=\frac{\partial \tilde{f}}{\partial \tau_{N}}\left(P, F\left(P, \tau_{N}\right), \tau_{N}\right)=0 .
$$

Now $\Phi$, being an element of $C^{\omega}\left(O_{1}^{*} \times I_{1}, \mathbb{R}\right)$, can be developed into a power series about the point $\left(P^{*}, \tau_{N}^{*}\right)$ :

$$
\Phi\left(P, \tau_{N}\right)=\sum_{\alpha \in \mathbb{N}_{0}^{3 N+9}} \sum_{j=0}^{\infty} \Phi_{\alpha, j}\left(P-P^{*}\right)^{\alpha}\left(\tau_{N}-\tau_{N}^{*}\right)^{j}
$$

for any pair $\left(P, \tau_{N}\right) \in O_{1}^{*} \times I_{1}$. Now we prove the existence of some positive integer $q$ with

$$
\Phi_{0,0}=\Phi_{0,1}=\cdots=\Phi_{0, q-1}=0, \text { but } \Phi_{0, q} \neq 0 .
$$

First, we note that there holds in particular

$$
\Phi_{0,0}=\Phi\left(P^{*}, \tau_{N}^{*}\right)=\frac{\partial \tilde{f}}{\partial \tau_{N}}\left(P^{*}, F\left(P^{*}, \tau_{N}^{*}\right), \tau_{N}^{*}\right)=0
$$

due to $\left(F\left(P^{*}, \tau_{N}^{*}\right), \tau_{N}^{*}\right)=\left(\hat{\tau}^{*}, \tau_{N}^{*}\right) \in K\left(\tilde{f}\left(P^{*}, \cdot\right)\right)$. But on the other hand if $\Phi_{0, j}$ would vanish for any $j \in \mathbb{N}_{0}$, then we could conclude that $\Phi\left(P^{*}, \cdot\right) \equiv 0$ on $I_{1}$, which would imply together with (30) that $\left\{\left(F\left(P^{*}, \tau_{N}\right), \tau_{N}\right) \mid \tau_{N} \in I_{1}\right\}$ would be an analytic curve of 
critical points of $\tilde{f}\left(P^{*}, \cdot\right)$ passing through the point $\left(F\left(P^{*}, \tau_{N}^{*}\right), \tau_{N}^{*}\right)=\tau^{*}$ which is contained in $K_{S}\left(f\left(P^{*}, \cdot\right)\right)$. But this is exactly the hypothesis at the beginning of Sect. 6 in [14] which is shown to lead to a contradiction in the course of Sects. 6 and 7 in [14] in combination with its improvement in the above section, in order to prove the finiteness of the set $K_{s}\left(f\left(P^{*}, \cdot\right)\right) \cong \mathcal{M}_{S}\left(\Gamma^{*}\right)$. Thus, there exists in fact some integer $q \geq 1$ satisfying the assertion in (32). Hence, all requirements of Weierstrass' preparation theorem (see [15], p. 152) are satisfied which yields the existence of some neighborhood $O_{2}^{*} \times I_{2}$ of $\left(P^{*}, \tau_{N}^{*}\right)$, of some Weierstrass polynomial

$$
W\left(P, \tau_{N}\right)=\left(\tau_{N}-\tau_{N}^{*}\right)^{q}+a_{q-1}(P)\left(\tau_{N}-\tau_{N}^{*}\right)^{q-1}+\cdots+a_{1}(P)\left(\tau_{N}-\tau_{N}^{*}\right)+a_{0}(P),
$$

defined on $O_{2}^{*} \times I_{2}$, with coefficients $a_{j} \in C^{\omega}\left(O_{2}^{*}, \mathbb{R}\right)$ satisfying $a_{j}\left(P^{*}\right)=0$, for $j=$ $0, \ldots, q-1$, and of some non-vanishing function $V \in C^{\omega}\left(O_{2}^{*} \times I_{2}, \mathbb{R}\right)$ which fulfill

$$
\Phi\left(P, \tau_{N}\right)=\frac{\Phi_{0, q}}{V\left(P, \tau_{N}\right)} W\left(P, \tau_{N}\right) \quad \forall\left(P, \tau_{N}\right) \in O_{2}^{*} \times I_{2}
$$

Thus, the zeroes of $\Phi(P, \cdot)$ and $W(P, \cdot)$ coincide on $I_{2}$ and their number is bounded by the degree $q \equiv q\left(P^{*}, \tau^{*}\right)$ of $W(P, \cdot)$ uniformly for any fixed $P \in O_{2}^{*}$. Thus together with Corollary $2.1,(31)$ and $I_{2} \subset I_{1}$ we infer that

$$
\begin{aligned}
& \sharp\left(K_{S}(f(P, \cdot)) \cap\left(\hat{U}_{1} \times I_{2}\right)\right) \leq \sharp\left(K(\tilde{f}(P, \cdot)) \cap\left(\hat{U}_{1} \times I_{2}\right)\right) \\
& \quad=\text { number of zeroes of } \Phi(P, \cdot) \text { on } I_{2} \leq q\left(P^{*}, \tau^{*}\right) \quad \forall P \in O_{2}^{*} .
\end{aligned}
$$

Now, we know that $K_{s}\left(f\left(P^{*}, \cdot\right)\right)(\neq \emptyset)$ consists of only finitely many points $\left\{\tau^{j}\right\}_{j=1, \ldots, J}$ that satisfy Case 1 or 2 , thus that possess disjoint open neighborhoods $U^{j}$ in $T$ for which there hold:

$$
\sharp\left(K_{S}(f(P, \cdot)) \cap U^{j}\right) \leq q^{j}\left(P^{*}, \tau^{j}\right) \quad \forall P \in O^{j},
$$

for $j=1, \ldots, J=\sharp\left(K_{S}\left(f\left(P^{*}, \cdot\right)\right)\right)$, where $q^{j}$ are degrees of Weierstrass polynomials depending on $P^{*}$ and $\tau^{j}$ only and where $O^{j}$ are sufficiently small neighborhoods of $P^{*}$ in $\mathbb{R}^{3 N+9}$. As the disjoint union $\bigcup_{j=1}^{J} U^{j}=: U$ is an open neighborhood of $K_{S}\left(f\left(P^{*}, \cdot\right)\right)$ in $T$ the above lemma yields the existence of some neighborhood $O$ of $P^{*}$ in $\mathbb{R}^{3 N+9}$ such that $K_{S}(f(P, \cdot)) \subset U \forall P \in O$, i.e. we have:

$$
K_{s}(f(P, \cdot))=\bigcup_{j=1}^{0} K_{s}(f(P, \cdot)) \cap U^{j} \quad \forall P \in O .
$$

Now choosing this neighborhood $O$ of $P^{*}$ sufficiently small such that there holds also $O \subset \bigcap_{j=1}^{J} O^{j}$ we obtain by (34) and (35):

$$
\begin{aligned}
\sharp\left(K_{S}(f(P, \cdot))\right) & =\sum_{j=1}^{J} \sharp\left(K_{S}(f(P, \cdot)) \cap U^{j}\right) \\
& \leq \sum_{j=1}^{J} q^{j}\left(P^{*}, \tau^{j}\right)=: \beta\left(P^{*}\right) \quad \forall P \in O .
\end{aligned}
$$

Thus, together with the correspondences $K_{S}(f(P, \cdot)) \cong \mathcal{M}_{S}(\Gamma(P))$ via $\psi(P, \cdot)$ and $P \longleftrightarrow \Gamma(P)$ we finally obtain the claimed result. 
Acknowledgments The author was supported by a research stipend of the Deutsche Forschungsgemeinschaft and would like to thank Prof. Ph.D. Tromba and Prof. Dr. Dierkes for their interest and hospitality and Prof. Dr. Hildebrandt for his tremendous support.

\section{References}

1. Alt, H.W.: Verzweigungspunkte von H-Flächen I. Math. Z. 127, 333-362 (1972)

2. Alt, H.W.: Verzweigungspunkte von H-Flächen II. Math. Ann. 201, 33-55 (1973)

3. Courant, R.: Critical points and unstable minimal surfaces. Proc. Natl. Acad. Sci. 27, $51-57$ (1941)

4. Dieudonné, J.: Foundations of Modern Analysis. Academic Press, New York (1960)

5. Heinz, E.: Über die analytische Abhängigkeit der Lösungen eines linearen elliptischen Randwert problems von den Parametern. Nachr. Akad. Wiss. Gött., Math.-Phys. K1.II, 1-20 (1979)

6. Heinz, E.: Über eine Verallgemeinerung des Plateauschen Problems. Manuscripta Math. 28, 81-88 (1979)

7. Heinz, E.: Ein mit der Theorie der Minimalflächen zusammenhängendes Variations problem. Nachr. Akad. Wiss. Gött., Math.-Phys. K1.II, 25-35 (1980)

8. Heinz, E.: Zum Marx-Shiffmanschen Variations problem. J. Reine u. Angew. Math. 344, 196-200 (1983)

9. Heinz, E.: Minimalflächen mit polygonalem Rand. Math. Z. 183, 547-564 (1983)

10. Hildebrandt, S., von der Mosel, H.: On two-dimensional parametric variational problems. Calc. Var. 9, 249-267 (1999)

11. Jakob, R.: H-surface-index-formula. I.H.P. Anal. Non-Lineaire 22, 557-578 (2005)

12. Jakob, R.: Schwarz operators of minimal surfaces spanning polygonal boundary curves. Accepted by Calc. Var., DOI: 10.1007/s00526-007-0098-5

13. Jakob, R.: Finiteness of the set of solutions of Plateau's problem with polygonal boundary curves. Bonner Math. Schriften 379, 1-95 (2006)

14. Jakob, R.: Finiteness of the set of solutions of Plateau's problem for polygonal boundary curves. Accepted by I.H.P. Anal. Non-lineaire, DOI: 10.1016/j.anihpc.2006.10.003

15. Krantz, S.: A Primer of Real Analytic Functions. Birkhäuser, Basel (1992)

16. Nitsche, J.C.C.: Vorlesungen über Minimalflächen. Grundlehren. Vol. 199. Springer, BerlinHeidelberg-New York (1975)

17. Sauvigny, F.: On the total number of branch points of quasi-minimal surfaces bounded by a polygon. Analysis 8, 297-304 (1988)

18. Sauvigny, F.: On immersions of constant mean curvature: Compactness results and finiteness theorems for Plateau's Problem. Arch. Rat. Mech. Anal. 110, 125-140 (1990)

19. Tomi, F.: On the Finite Solvability of Plateau's Problem. Springer Lecture Notes in Math. 579, 679695 (1977) 